\title{
Lewis antigen-negative pancreatic cancer: An aggressive subgroup
}

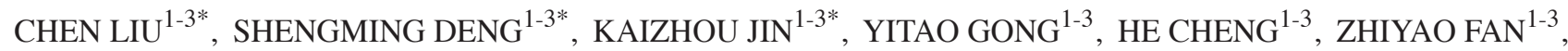 \\ YUNZHEN QIAN $^{1-3}$, QIUYI HUANG ${ }^{1-3}$, QUANXING NI ${ }^{1-3}$, GUOPEI LUO $^{1-3}$ and XIANJUN YU ${ }^{1-3}$ \\ ${ }^{1}$ Department of Pancreatic Surgery, Fudan University Shanghai Cancer Center; \\ ${ }^{2}$ Department of Oncology, Shanghai Medical College, Fudan University; ${ }^{3}$ Pancreatic Cancer Institute, \\ Fudan University, Shanghai Pancreatic Cancer Institute, Shanghai 200032, P.R. China
}

Received December 26, 2019; Accepted January 7, 2020

DOI: 10.3892/ijo.2020.4989

\begin{abstract}
Carbohydrate antigen 19-9 (CA19-9) is the most important biomarker for pancreatic cancer. Approximately $5-10 \%$ of individuals are Lewis antigen negative with scarce secretion of CA19-9 and fucosylation deficiency. However, the characteristics of Lewis-negative pancreatic cancer are unidentified. Clinicopathological characteristics of 853 patients with pancreatic cancer were examined. Pancreatic cancer cell lines were sequenced for Lewis status. Morphological and molecular features of pancreatic cancer cells were compared. Orthotopic animal modes were established. Lewis-negative patients had poorer outcome $(\mathrm{P}<0.001)$, higher metastatic rate $(\mathrm{P}=0.004)$, lower CA19-9 expression $(\mathrm{P}<0.001)$ and higher MUC16 expression $(\mathrm{P}<0.001)$ than Lewis-positive patients. Lewis-negative cells (CaPan-1, MiaPaCa-2 and Panc-1) showed a shuttle shape with scarce pseudopods. Overall, Lewis-negative cells had higher proliferation rate, higher migration ability, lower fucosylation, lower CA19-9 expression and higher MUC16 expression than Lewis-positive cells (BxPC-3, SU8686, SW1990). Lewis-negative cell line MiaPaCa-2 corresponded to larger orthotopic tumor than Lewis-positive cells SU8686. Potential proteoglycans were identified in Lewis-positive cancer, including EGFR, HSPG2, ADAM17, GPC1, ITGA2, CD40, IL6ST and GGT1. Therefore, Lewis-negative pancreatic cancer is an aggressive subgroup with special clinical and molecular features.
\end{abstract}

\section{Introduction}

Pancreatic cancer is one of the most lethal malignancies in the world, with its mortality close to its incidence $(1,2)$. In recent

Correspondence to: Dr Xianjun Yu or Dr Guopei Luo, Department of Pancreatic Surgery, Fudan University Shanghai Cancer Center, 270 Dong'An Road, Xuhui, Shanghai 200032, P.R. China

E-mail: yuxianjun@fudanpci.org

E-mail: luoguopei@hotmail.com

*Contributed equally

Key words: pancreatic adenocarcinoma, CA19-9, FUT3, fucosyltransferase, CA125 years, the incidence of pancreatic cancer keeps rising due to the popularization of the westernized lifestyle (3). Approximately $80 \%$ of patients with pancreatic cancer are diagnosed at an advanced stage and miss the chance for curative resection (2). Pancreatic cancer, as a highly heterogenous tumor, is a major clinical challenge $(2,4,5)$. Therefore, identifying subgroups with special biology is urgently needed for the management of pancreatic cancer.

Carbohydrate antigen 19-9 (CA19-9), also called sialyl Lewis antigen $\mathrm{A}$, is the most important biomarker for pancreatic cancer (6-8). The sensitivity in detecting pancreatic cancer is $\sim 80 \%$ for CA19-9 (9). In the population, $\sim 5-10 \%$ of individuals are Lewis antigen negative, with no or low secretion of CA19-9 (10). In a previous study, we showed that Lewis-negative patients had poorer outcome than Lewis-positive patients (11). Fucosyltransferase 3 (also called Lewis gene), an $\alpha 1,3 / 4$-fucosyltransferase, is the key enzyme of CA19-9 biosynthesis and plays a critical role in protein fucosylation (12). Protein fucosylation has undoubtedly an important effect on the function of proteins, and affects cancer development (12). Therefore, Lewis-negative pancreatic cancer, which is deficient in fucosylation, may have a special biology, different from Lewis-positive cancer (11). However, the characteristics of Lewis-negative pancreatic cancer are largely unidentified.

In the present study, the characteristics of Lewis-negative pancreatic cancer in both clinical findings and basic research were investigated. The clinicopathological characteristics of 853 patients with pancreatic cancer classified by Lewis status were examined. Six pancreatic cancer cell lines were sequenced to determine their Lewis status. Morphological and molecular features of pancreatic cancer cells classified by Lewis status were compared. An orthotopic tumor model was constructed.

\section{Materials and methods}

Patients and data collection. Medical data were retrieved from a prospectively maintained database of the Fudan University Shanghai Cancer Center (Shanghai, China) from September 2004 to November 2011. Data including age, sex, tumor location, metastasis, grade, CA19-9, carbohydrate antigen 125 (CA125, also called MUC16), nerve invasion, lymphovascular invasion, and lymphatic metastasis were retrieved. The primary endpoint was overall survival and follow-up data were updated till October 2019. The study 
protocol was authorized by the Ethics Committee of the Fudan University Shanghai Cancer Center. Written informed consent was acquired from all of the patients enrolled in the study.

Immunohistochemistry. Tissues were fixed in $10 \%$ formalin for $12 \mathrm{~h}$ at room temperature. Formalin-fixed, paraffin-embedded sections $(4 \mu \mathrm{m})$ of surgically resected pancreatic cancer tissues were obtained [20 cases of Lewis (-), 19 cases of Lewis (+)]. After tissue sections were deparaffinized with xylene, the endogenous peroxidase activity was blocked with $3 \% \mathrm{H}_{2} \mathrm{O}_{2}$ in methanol at $37^{\circ} \mathrm{C}$ for $20 \mathrm{~min}$. Sections were incubated with specific primary antibodies against MUC16 (1:200, cat. no. 60261-1-Ig; ProteinTech Group, Inc.) overnight at $4^{\circ} \mathrm{C}$. The antibody solution was removed, and the sections were washed in wash buffer 3 times for $10 \mathrm{~min}$ each. Secondary antibody (GTVision III immunohistochemical detection kit, GK5005; Gene Tech Co., Ltd.) was added to each section and the tissues were incubated for $1 \mathrm{~h}$ at room temperature. An avidin-biotin-peroxidase complex solution was used for the visualization of immunoreactions using 3,3'-diaminobenzidine to detect the protein-antibody complexes. Protein expression levels were classified as positive and negative staining using an optical microscope with a magnification of 1:400.

Lewis genotyping. Lewis status was determined by Sanger sequencing using genomic DNA extracted from blood specimens or pancreatic cancer cell lines, as previously described $(9,11,13)$. In order to detect variants in the Lewis gene: T59G, T202C, C314T, G508A and T1067A, the following primers were used for polymerase chain reaction amplification: $358 \mathrm{~F}$, GGGTGCAGC CAAGCCACAA and 358R, AGGTGGGAGGCGTGACTT AGG; P1F, ACTTGGAGCCACCCCCTAACTGCCA and 508R, CGGCCTCTCAGGTGAACCAAGCCGCT).

Cell lines. BxPC-3, SU8686, SW1990, CaPan-1, MiaPaCa-2 and Panc-1 human pancreatic cancer cells were kindly provided by Stem Cell Bank, Chinese Academy of Sciences (Shanghai, China). The CaPan-1, MiaPaCa-2, Panc-1 and SW1990 cells were kept in DMEM supplemented with 10\% FBS and $1 \%$ penicillin/streptomycin (Gibco; Thermo Fisher Scientific, Inc.). The BxPC-3 and SU8686 cells were kept in RPMI-1640 medium supplemented with $10 \%$ FBS and penicillin/streptomycin (Gibco; Thermo Fisher Scientific, Inc.). All cells were maintained at humidified incubator at a $37^{\circ} \mathrm{C}$ with $5 \% \mathrm{CO}_{2}$. Only mycoplasma-negative cells were used for the experiments.

Phase-contrast microscopy and scanning electron microscopy. Human pancreatic cancer cells were seeded into $10-\mathrm{cm}$ dishes and images were captured by a phase-contrast microscope (Leica Microsystems GmbH). For the FEI Quanta 200 scanning electron microscope (Philips Healthcare), the cells were seeded into $0.8-\mathrm{cm}$ glass slides treated with polylysine amino acid coating by gold powder. The cells were fixed in $2.5 \%$ glutaraldehyde solution at $4^{\circ} \mathrm{C}$ for $5 \mathrm{~h}$. After being washed with $0.1 \mathrm{~mol} / 1$ phosphoric acid buffer 3 times, the cells were dehydrated by alcohol step by step, replaced by pure alcohol, dried at the critical point of carbon dioxide, and then observed and photographed by FEI Quanta 200 scanning electron microscope after coating. All images were captured by random fields.
Cell proliferation assay. For cell proliferation, the pancreatic cancer cells were trypsinized, and $3 \times 10^{3}$ cells were seeded into 96-well plates (Corning, Inc.). After certain culture periods, $10 \mu \mathrm{l}$ of Cell Counting Kit-8 (CCK-8; Dojindo Molecular Technologies, Inc.) were added into the wells and the cells were incubated at a humidified incubator at $37^{\circ} \mathrm{C}$ with $5 \% \mathrm{CO}_{2}$. Absorbance was detected on a multifunctional microplate reader at a wavelength of $450 \mathrm{~nm}$.

Transwell migration assay. Pancreatic cancer cells were trypsinized, and $3 \times 10^{4}$ cells were seeded into Transwell inserts ( $8.0 \mathrm{~mm}$ pore; BD Falcon; BD Biosciences) without serum. FBS (10\%) and penicillin/streptomycin (Gibco; Thermo Fisher Scientific, Inc.) were plated into the lower chamber. After $24 \mathrm{~h}$, the upper side of the membrane was rubbed with cotton swap and the cells were fixed in $4 \%$ paraformaldehyde and stained by $0.3 \%$ crystal violet for $20 \mathrm{~min}$ at room temperature. After crystal violet staining, the number of cells migrating to the basal side insert was counted. Stained cells were counted in seven randomly selected fields using an optical microscope with a magnification of 1:400.

Western blot and lectin blot analyses. Human cells were seeded into60- $\mathrm{mm}^{2}$ dishes. Cells were harvested and lysed in RIPAbuffer, $1 \mathrm{mM}$ PMSF and 1X Protease Inhibitor Cocktail (Beyotime Institute of Biotechnology) for $30 \mathrm{~min}$. Protein concentration was determined by a BCA assay. The protein samples $(10 \mu \mathrm{g})$ were loaded on a $10 \%$ SDS-PAGE and run at $100 \mathrm{~V}$ for $80 \mathrm{~min}$ in 1X SDS-PAGE running buffer (25 mM Tris, $192 \mathrm{mM}$ glycine, $1 \%$ SDS). Proteins were transferred onto a $0.45-\mathrm{mm}$ nitrocellulose membrane (EMD Millipore) using a wet transfer protocol with 1X transfer buffer (25 mM Tris, $192 \mathrm{mM}$ Glycine, 20\% methanol) at $300 \mathrm{~mA}$ for $110 \mathrm{~min}$ at Mini Trans-Blot Cell Module (Bio-Rad Laboratories, Inc.) in ice box. The membranes were blocked with PBST (0.02\% Tween-20 in PBS) containing $5 \%$ skim milk (BD Biosciences) at room temperature. The membranes were incubated with anti-MUC16 antibody $(1: 1,000$, cat. no. 20077-1-AP; ProteinTech Group, Inc.), anti-EGFR antibody (1:1,000, cat. no. ab52894; Abcam), anti-STAT3 antibody (1:1,000, cat. no. ab32143; Abcam) and HRP-conjugated $\beta$-actin antibody (1:5,000, cat. no. HRP-60008; ProteinTech Group, Inc.) in blocking solution on a shaker at $4^{\circ} \mathrm{C}$ overnight. Following the primary incubation, the membranes were incubated with goat-anti-mouse $\operatorname{IgG}(\mathrm{H}+\mathrm{L})$-HRP or goat-anti-rabbit IgG (H+L)-HRP (cat. no. SA00001-2; ProteinTech Group, Inc.) at 1:5,000 in PBST on a shaker for $1 \mathrm{~h}$ at room temperature. For lectin blot analysis, biotinylated Aleuria aurantia lentin (AAL, $3 \mu \mathrm{g} / \mathrm{ml}$ ) (Vector Laboratories, Inc.) was incubated with $3 \%$ bovine serum albumin on a shaker for $30 \mathrm{~min}$, and then, incubated with $0.1 \mu \mathrm{g} / \mathrm{ml}$ streptavidin-HRP conjugate (Vector Laboratories, Inc.) in blocking buffer for $20 \mathrm{~min}$ at room temperature. Images were captured after SuperSignal West Femto ECL (BR11121; Bridgen Co., Ltd.) reaction.

Liquid chromatography-mass spectrometry (LC-MS) for protein glycosylation. Pancreatic cancer cells ( $>2 \times 10^{7}$ cells) were freshly prepared prior to use. The sample proteins were extracted using SDT lysis buffer (4\% SDS, 100 mM DTT, $100 \mathrm{mM}$ Tris-HCl pH 8.0). Samples were boiled for $3 \mathrm{~min}$ and further ultrasonicated. Undissolved beads were 
Table I. Baseline characteristics of patients with pancreatic cancer classified by Lewis status.

\begin{tabular}{|c|c|c|c|c|}
\hline Characteristics & Total & Lewis positive & Lewis negative & P-value \\
\hline No. of cases & 853 & 753 & 100 & \\
\hline Median survival (months) & 12.6 & 13.3 & 7.4 & $<0.001$ \\
\hline Age (years) & & & & 0.867 \\
\hline$\leq 60$ & 382 & 338 & 44 & \\
\hline$>60$ & 471 & 415 & 56 & \\
\hline Sex & & & & 0.923 \\
\hline Male & 508 & 448 & 60 & \\
\hline Female & 345 & 305 & 40 & \\
\hline Location $^{\mathrm{a}}$ & & & & 0.582 \\
\hline Head & 431 & 383 & 48 & \\
\hline Others & 421 & 369 & 52 & \\
\hline CA19-9 (U/ml) & $453.5 \pm 617.0$ & $499.7 \pm 635.0$ & $106.0 \pm 273.1$ & $<0.001$ \\
\hline CA125 (U/ml) & $149.4 \pm 437.7$ & $135.8 \pm 401.6$ & $251.9 \pm 642.0$ & $<0.001$ \\
\hline Metastasis & & & & 0.004 \\
\hline Yes & 314 & 264 & 50 & \\
\hline No & 539 & 489 & 50 & \\
\hline Grade $^{\mathrm{b}}$ & & & & 0.245 \\
\hline High, medium & 235 & 219 & 16 & \\
\hline Low & 148 & 133 & 15 & \\
\hline Nerve invasion ${ }^{\mathrm{b}}$ & & & & 0.259 \\
\hline Yes & 318 & 290 & 28 & \\
\hline No & 65 & 62 & 3 & \\
\hline Lymphovascular invasion $^{\mathrm{b}}$ & & & & 0.934 \\
\hline Yes & 84 & 77 & 7 & \\
\hline No & 298 & 274 & 24 & \\
\hline Lymph involvement ${ }^{\mathrm{b}}$ & & & & 0.773 \\
\hline Yes & 164 & 150 & 14 & \\
\hline No & 220 & 203 & 17 & \\
\hline
\end{tabular}

${ }^{a}$ There was unknown information of tumor location for one patient. ${ }^{b}$ For curative resected cases only. CA19-9, carbohydrate antigen 19-9; CA125, carbohydrate antigen 125 .

removed by centrifugation at $16,000 \mathrm{x} \mathrm{g}$ for $15 \mathrm{~min}$. The supernatant containing proteins was collected. Protein digestion was performed with FASP method, as described by Wiśniewski et al (14). Proteins were subjected to glycopeptide enrichment and were deglycosylated. Eluted peptides were collected and dried for further LC-MS analysis (Thermo Fisher Scientific, Inc.) using a positive or negative ionization mode.

Reverse-phase high-performance liquid chromatography separation was performed with the EASY-nLC system (Thermo Fisher Scientific, Inc.) using a self-packed column (75 $\mu \mathrm{m}$ x $150 \mathrm{~mm} ; 3 \mu \mathrm{m}$ ReproSil-Pur C18 beads, $120 \AA$; Dr. Maisch GmbH HPLC) at a flow rate of $300 \mathrm{nl} / \mathrm{min}$. MS data were acquired using a data-dependent top 20 method dynamically choosing the most abundant precursor ions from the survey scan $(300-1,800 \mathrm{~m} / \mathrm{z})$ for HCD fragmentation. The instrument was run with peptide recognition mode enabled. A lock mass of 445.120025 Da was used as internal standard for mass calibration. The full MS scans were

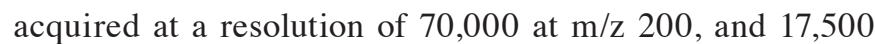
at $\mathrm{m} / \mathrm{z} 200$ for MS/MS scan. MS data were analyzed using
MaxQuant software (version 1.6.1.0; Max Planck Institute of Biochemistry) and were searched against the SwissProt human database (http://www.expasy.ch/sprot/).

Bioinformatics analyses were carried out with Perseus software (Max Planck Institute of Biochemistry), Microsoft Excel (Microsoft Corporation) and R statistical computing software (Free Software Foundation's GNU General Public License; https://www.r-project.org/about.html). Construction of protein-protein interaction networks was conducted using the STRING database (https://string-db.org) with Cytoscape software (an open source software platform provided by the National Resource for Network Biology). The MS data were analyzed using MaxQuant software (version 1.6.1.0) and were searched against the SwissProt human database (20,431 total entries; downloaded, 10/15/2019). The Motif-X algorithm in the MEME Suite (version 5.1.0) was used for N-linked glycosylation motif analysis.

Orthotopic animal model. A total of 16 male BALB/c nude (nu/nu) mice (6-8 weeks of age) were purchased from Shanghai 


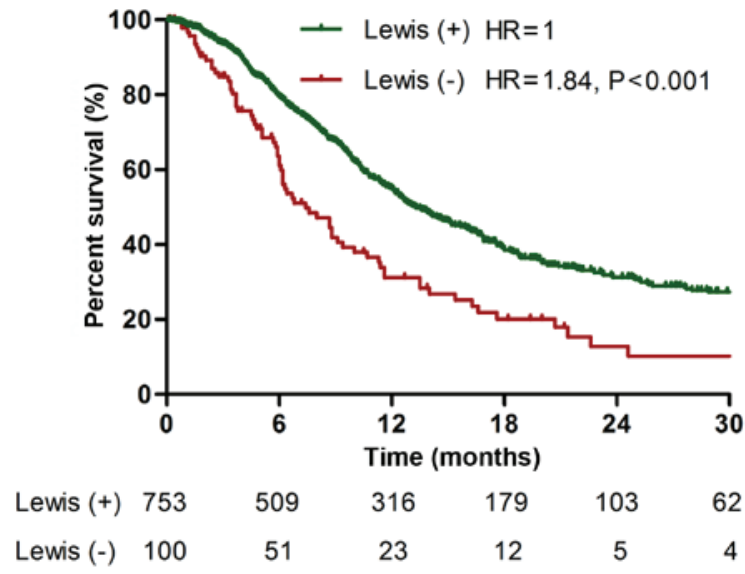

Figure 1. Kaplan-Meier survival curves of patients with pancreatic cancer classified by Lewis status. Lewis-negative patients $(n=100)$ had poorer prognosis than Lewis-positive patients $(\mathrm{n}=753, \mathrm{P}<0.001)$.

SLAC Laboratory Animal Co., Ltd. and 8 mice were included in each group. Animals were housed in laminar flow cabinets under specific pathogen-free conditions. The housing conditions were as follows: temperature $22 \pm 1^{\circ} \mathrm{C}$, humidity $50 \%$, 12-h dark/light cycle, and ad libitum access to food and water. Animals were orthotopically injected with $1 \times 10^{6} / \mathrm{ml}$ cells into the pancreas $(n=8)$. The mice were sacrificed at 5 -week endpoints to examine tumor weight. Histological features of tumors were examined by hematoxylin and eosin (H\&E; Beyotime Institute of Biotechnology) staining. All mouse samples were fixed with $10 \%$ buffer formalin at room temperature (24-36 h) to make formalin-fixed, paraffin-embedded tissue blocks. H\&E staining was performed on 3-mm thick sections at room temperature for $10 \mathrm{~min}$. The staining was observed by a light microscope (CKX41; Olympus Corporation), with a magnification of $\mathrm{x} 100$. All animal procedures were approved by the Institutional Animal Care Committee of Fudan University (Shanghai, China).

Statistical analysis. SPSS 19.0 software (IBM Corp.) and Prism statistical software (version 8; GraphPad Software, Inc.) were used for the statistical analysis of the data. Unpaired two-tailed Student's t-tests were used to determine the statistical differences between two groups. Data were presented as the mean \pm standard error of the mean. Dichotomous variables were analyzed by Chi-square test or Fisher's exact test. Survival analysis was assessed by the Kaplan-Meier method and the survival curves were compared by log-rank tests. $\mathrm{P}<0.05$ was considered to indicate a statistically significant difference.

\section{Results}

Clinicopathological characteristics of Lewis-negative pancreatic cancer patients. A total of 853 patients with pancreatic cancer were included to undergo Lewis antigen evaluation and $11.7 \%$ of patients were Lewis negative (Table I). The median survival time of Lewis-negative patients was 7.4 months, which was significantly shorter than that of Lewis-positive patients (13.3 months, $\mathrm{P}<0.001$; Fig. 1). In addition, Lewis-negative patients had higher proportion of metastasis $(\mathrm{P}=0.004)$ than Lewis-positive patients. Lewis-negative patients had lower serum level of CA19-9 $(106.0 \pm 273.1 \mathrm{U} / \mathrm{ml})$ than Lewis-positive patients $(499.7 \pm 635.0 \mathrm{U} / \mathrm{ml}, \mathrm{P}<0.001)$. However, contrary to CA19-9, Lewis-negative pancreatic cancer secreted higher level of serum CA125 $(251.9 \pm 642.0 \mathrm{U} / \mathrm{ml})$ compared with Lewis-positive cancer $(135.8 \pm 401.6 \mathrm{U} / \mathrm{ml}, \mathrm{P}<0.001)$. These data show that Lewis-negative pancreatic cancer has aggressive clinicopathological characteristics with low secretion of CA19-9 and high secretion of CA125.

MUC16 expression in pancreatic cancer tissues. To confirm the association between Lewis status and CA125 secretion, the expression of MUC16 in pancreatic cancer tissues was detected by immunohistochemistry. Lewis-negative pancreatic cancer tissues (16/20) had higher levels of MUC16 expression than Lewis-positive cancer tissues $(9 / 19$, $\mathrm{P}=0.048$; Fig. 2).

Lewis antigen status of human pancreatic cancer cell lines. Sanger sequencing of the Lewis gene was carried out for the determination of the Lewis antigen status of human pancreatic cancer cell lines (BxPC-3, SU8686, SW1990, CaPan-1,

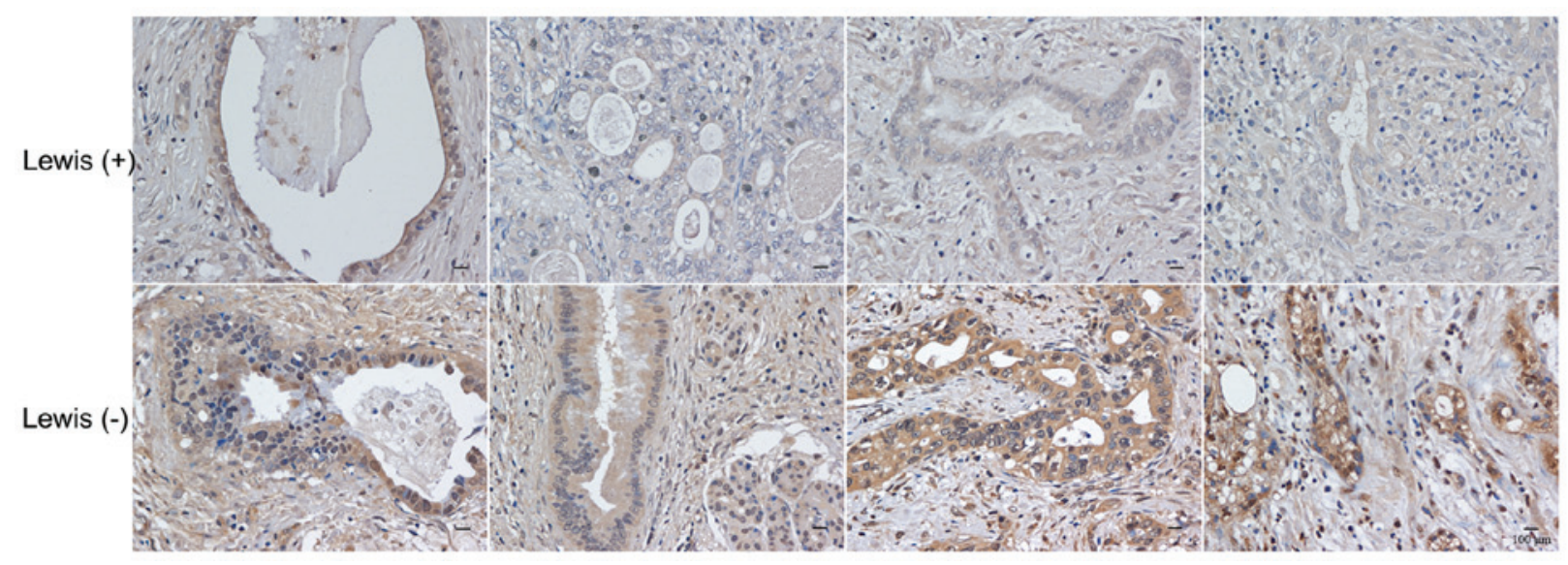

Figure 2. Immunohistochemical detection of MUC16 expression in pancreatic adenocarcinoma tissues. Lewis-negative pancreatic cancer tissues presented higher levels of MUC16 expression than Lewis-positive cancer tissues. 


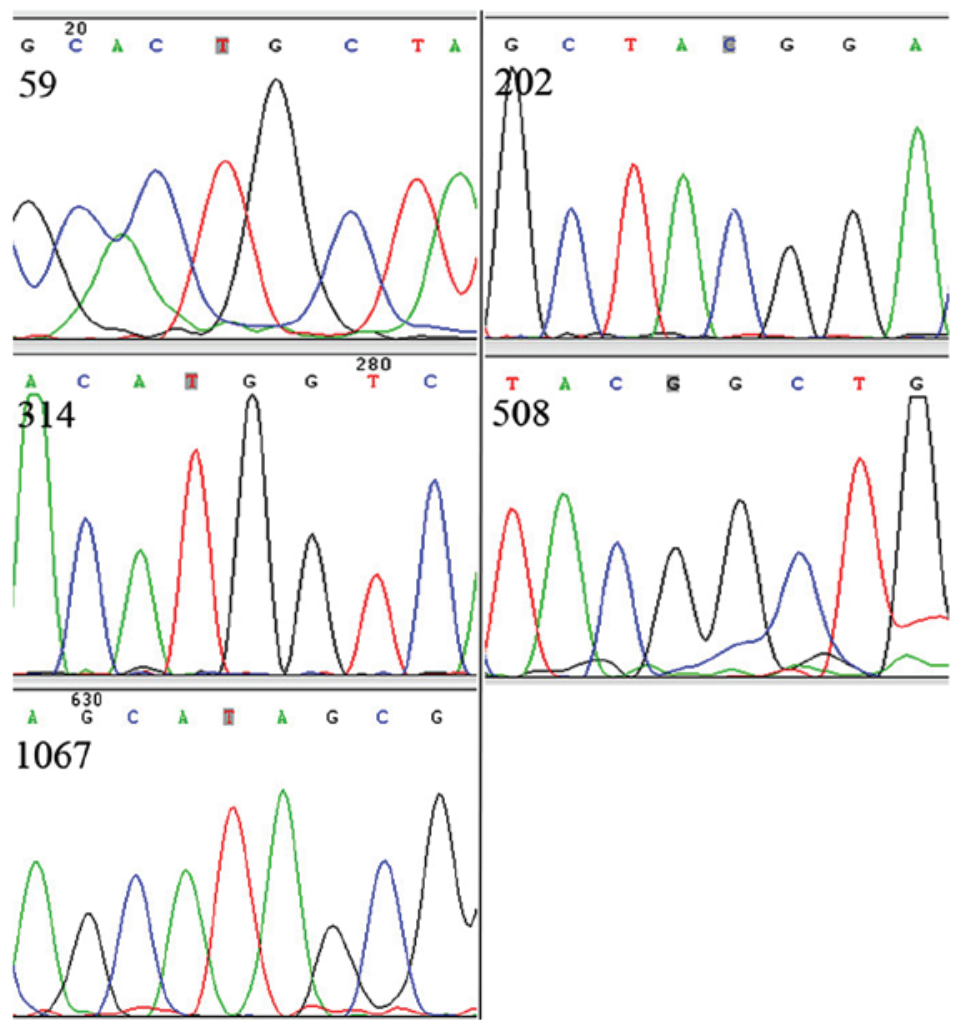

Figure 3. Represent sequencing results of the Lewis gene in the pancreatic cancer cell line Panc-1. The Panc-1 cell line had homozygous variations at 202 and 314 alleles and was classified as Lewis antigen negative.

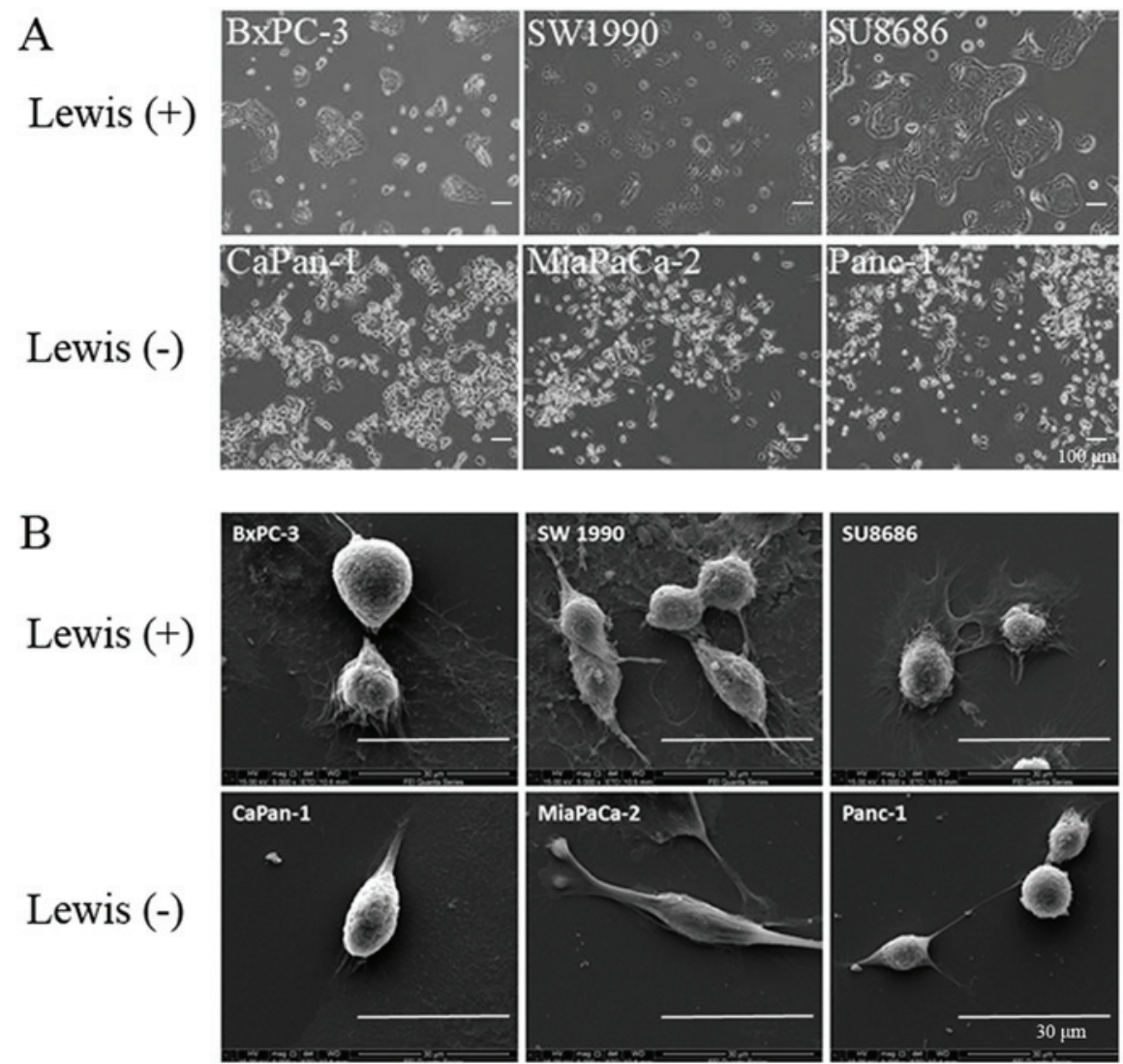

Figure 4. Morphology of pancreatic cancer cell lines classified by Lewis status. (A) Lewis-positive cells (BxPC-3, SW1990 and SU8686) grew in a cluster pattern, whereas Lewis-negative cells (CaPan-1, MiaPaCa-2 and Panc-1) showed a shuttle-like morphology, using phase-contrast microscopy. (B) Scanning electron microscopy showed that Lewis-positive cells were characterized by abundant pseudopods closely attached to the culture dish, whereas Lewis-negative cells were not. 


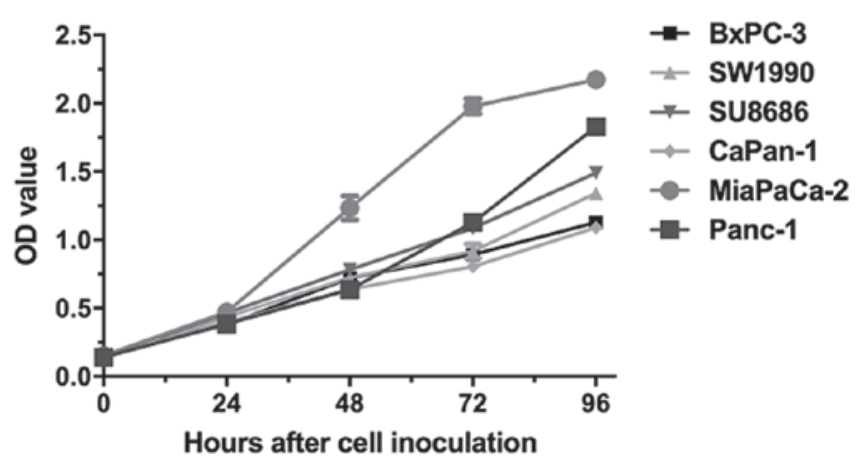

Figure 5. Proliferation of pancreatic cancer cell lines examined by CCK- 8 assay. Overall, Lewis-negative cells had significantly higher proliferation rate than Lewis-positive cells ( $\mathrm{P}=0.006)$. CCK-8, Cell Counting Kit-8.

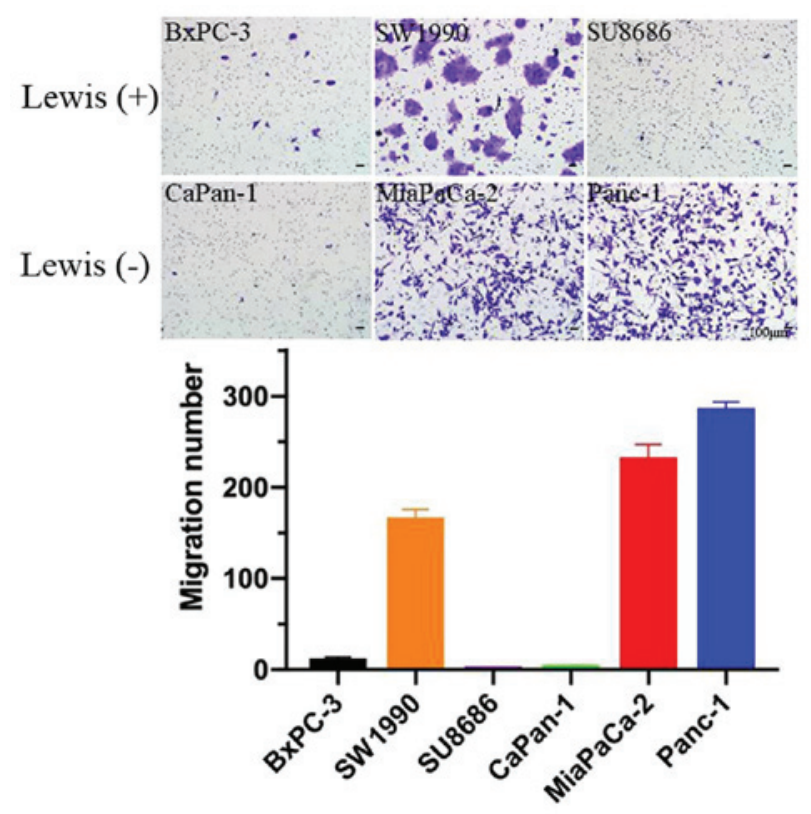

Figure 6. Migration ability of cancer cells examined via Transwell migration assay. Overall, Lewis-negative cells exhibited higher migration ability than Lewis-positive cells.

MiaPaCa-2 and Panc-1). Three cell lines were classified as Lewis positive (BxPC-3, SU8686 and SW1990) and the other three were categorized as Lewis negative (CaPan-1, MiaPaCa-2 and Panc-1). Representative sequencing results are shown in Fig. 3, which demonstrate homozygous mutations at 202 and 314 alleles of Lewis gene in the Panc-1 cell line.

Cell morphology. The difference in morphology between Lewis-positive and -negative cell lines was examined by phase-contrast microscopy and scanning electron microscopy. Lewis-positive cell lines (BxPC-3, SW1990 and SU8686) grew in a cluster pattern, whereas Lewis-negative cell lines (CaPan-1, MiaPaCa-2 and Panc-1) showed a shuttle-like morphology by phase-contrast microscopy (Fig. 4A). Scanning electron microscopy showed that Lewis-positive cells were characterized by abundant pseudopods closely attached to the culture dish, whereas Lewis-negative cells were not (Fig. 4B). Hence, these results suggest that there is a difference in cell morphology between different Lewis phenotype cells.

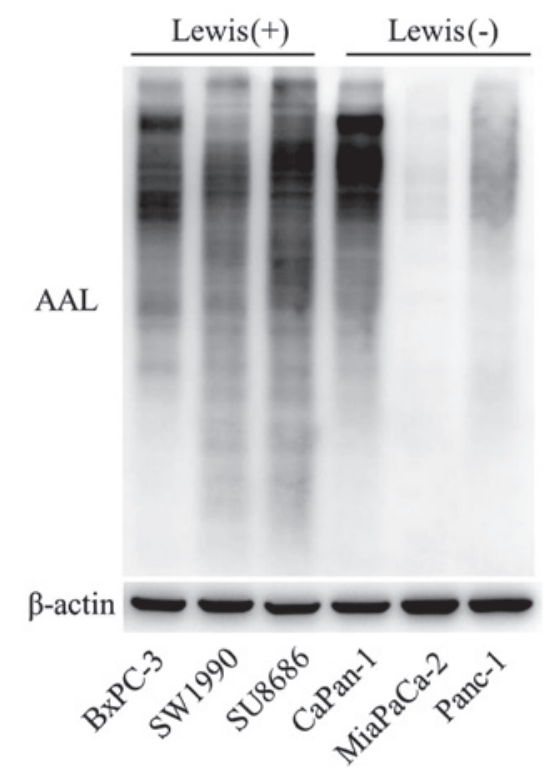

Figure 7. Levels of fucosylation evaluated by AAL examination. Lewis-negative cell lines (MiaPaCa-2 and Panc-1) demonstrated lower levels of fucosylation than Lewis-positive cell lines. ALL, Aleuria aurantia lentin.

Cell proliferation. The proliferative abilities of Lewis-positive and -negative cells were evaluated by CCK- 8 assay. Overall, Lewis-negative cells had significantly higher proliferation rate than Lewis-positive cells at $96 \mathrm{~h}$ after seeding $(\mathrm{P}=0.006$; Fig. 5). MiaPaCa-2, a Lewis-negative cell line, had the highest proliferation rate among all cells.

Cell migration. The migration ability of Lewis-positive and -negative cells was examined by Transwell assay. Approximately $3 \times 10^{4}$ pancreatic cancer cells were seeded into Transwell chambers and crystal violet staining was examined after $24 \mathrm{~h}$ of seeding. Overall, Lewis-negative cell lines exhibited higher migration ability compared with Lewis-positive cell lines ( $\mathrm{P}=0.003$; Fig. 6).

Level of fucosylation. The level of fucosylation in Lewis-positive and -negative cells was determined by AAL blotting analysis, which has been often used as carbohydrate probes for core fucose in glycoproteins. Lewis-negative cell lines (MiaPaCa-2 and Panc-1) exhibited lower levels of AAL compared with Lewis-positive cell lines (Fig. 7). This finding reveals that the lower fucosylation level may be attributed to the loss of function of the Lewis gene in Lewis-negative pancreatic cancer.

Glycoprotein and protein expression levels. According to clinical data, Lewis-negative patients had lower levels of serum CA19-9 than Lewis-positive patients (Table I). This result was further verified in pancreatic cancer cell lines. Western blot analysis revealed that the level of CA19-9 was significantly higher in Lewis-positive cells than that in Lewis-negative cells (Fig. 8). Lewis-negative cells displayed higher level of MUC16 compared with Lewis-positive cells. The association between MUC16 and Lewis status was consistent with the clinical results of CA125 and Lewis status. Differences in Lewis genotype had no significant effect on EGFR or STAT3 expression. 


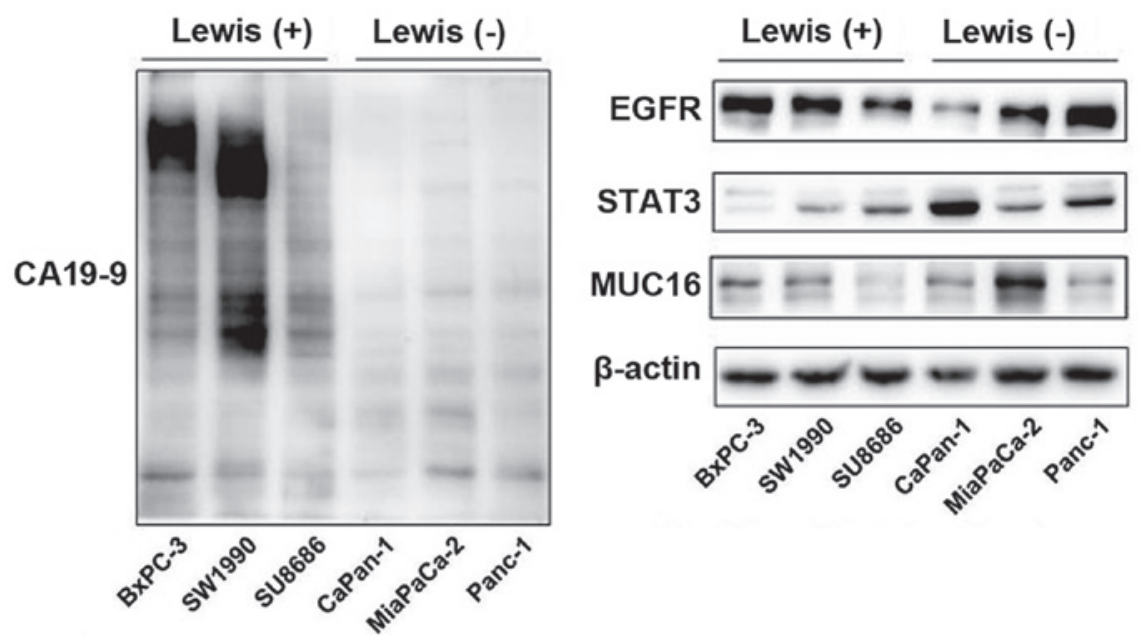

Figure 8. Glycoprotein and protein expression levels examined by western blot analysis. Lewis-negative cells displayed lower levels of CA19-9 and higher levels of MUC16 than Lewis-positive cells. CA19-9, carbohydrate antigen 19-9.

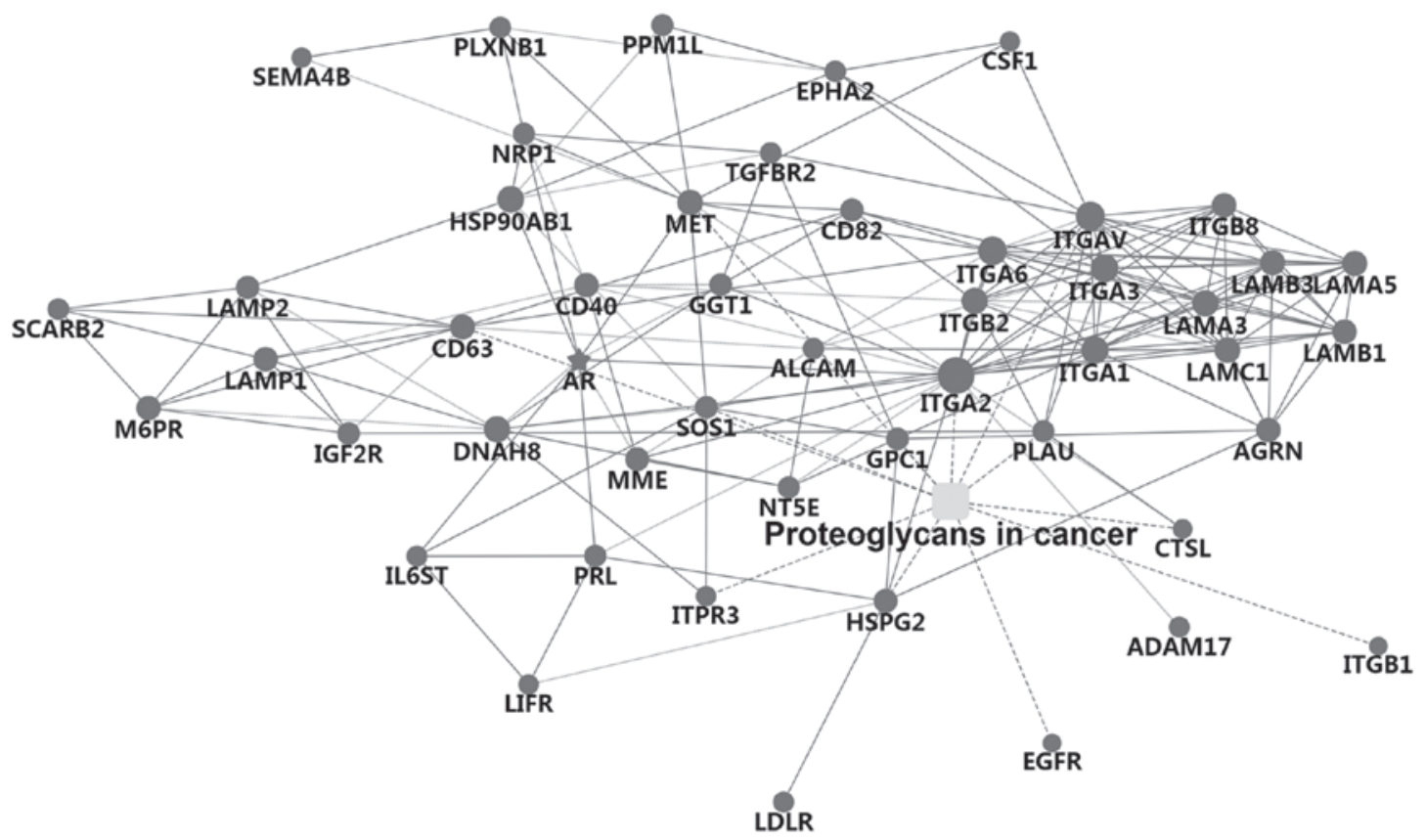

Figure 9. Network of cancer-related proteoglycans in the Lewis-positive cell line SU8686 detected by LC-MS. LC-MS, liquid chromatography-mass spectrometry.
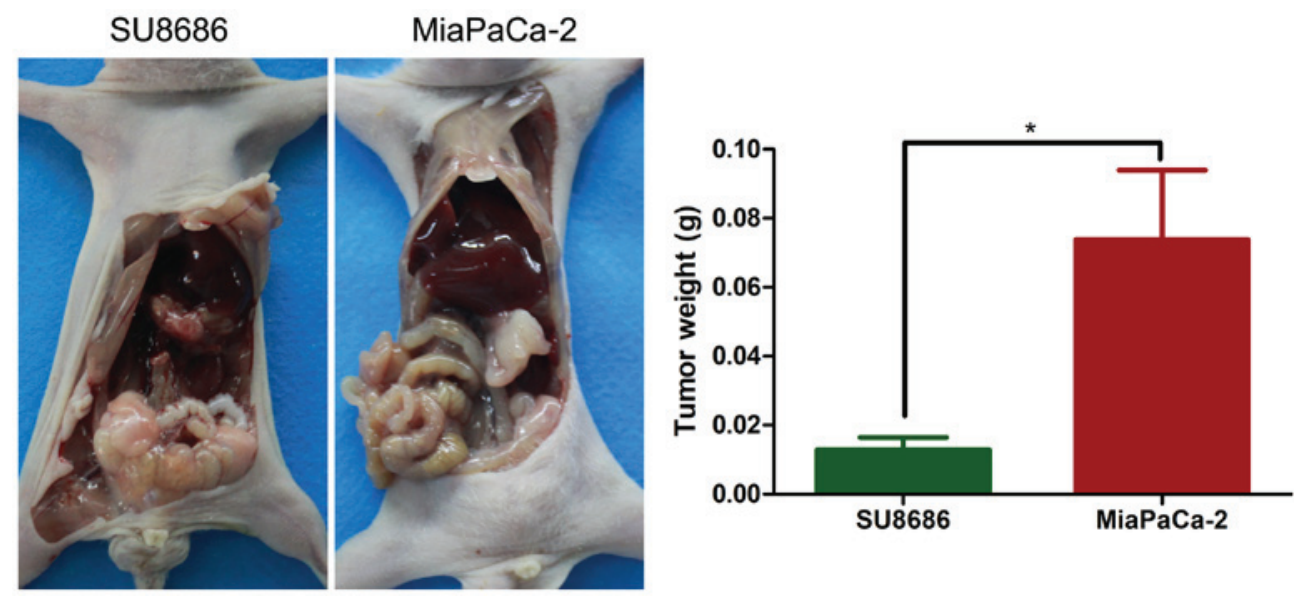

Figure 10. Orthotopic model of pancreatic cancer demonstrated that Lewis-negative pancreatic cancer cell line MiaPaCa-2 corresponded to higher tumor weight than Lewis-positive pancreatic cell line SU8686. ${ }^{*} \mathrm{P}=0.008$. 
Network of cancer-related proteoglycans. Lewis gene is a regulator of glycosylation and plays a key role in fucosylation of proteins. In order to further verify the role of the Lewis gene on fucosylation, cancer-related proteoglycans were detected by LC-MS in the Lewis-positive cell line SU8686 (Fig. 9). Potential proteoglycan interactions were identified, such as EGFR, HSPG2, ADAM17, GPC1, ITGA2, CD40, IL6ST and GGT1.

Orthotopic animal model. In order to examine the in vivo growth ability of pancreatic cancer cell lines classified by Lewis status, an orthotopic animal model was constructed by injection of tumor cells into the pancreas. Lewis-negative pancreatic cancer cell line $\mathrm{MiaPaCa}-2$ corresponded to higher tumor weight than Lewis-positive pancreatic cell line SU8686 (P=0.008; Fig. 10).

\section{Discussion}

Lewis gene is critical for fucosylation and protein modification (12). In the present study, a total of 853 patients with pancreatic cancer were included and $11.7 \%$ of patients were Lewis negative. Lewis-negative pancreatic cancer presented aggressive clinicopathological characteristics with low secretion of CA19-9 and high secretion of CA125. Three cell lines were classified as Lewis positive (BxPC-3, SU8686 and SW1990) and three were classified as Lewis negative (CaPan-1, MiaPaCa-2 and Panc-1). Lewis-negative pancreatic cancer cells had a shuttle shape with scarce pseudopods. Overall, Lewis-negative pancreatic cancer cells demonstrated higher proliferation rate, higher migration ability, lower fucosylation, lower expression of CA19-9 and higher expression of MUC16 than Lewis-positive cells. Potential proteoglycan interactions were identified by LC-MS, such as EGFR, HSPG2, ADAM17, GPC1, ITGA2, CD40, IL6ST and GGT1. These findings suggest that Lewis-negative pancreatic cancer is a unique and aggressive subgroup of pancreatic cancer with special clinical and molecular features.

CA19-9 is the most widely used biomarker in the management of pancreatic cancer $(6,11,15,16)$. Some studies have even reported that CA19-9 is not a bystander but an effector that could promote pancreatic cancer progression (17-19). CA19-9 activation could lead to the modification of fibulin-3, which hyperactivates EGFR signaling and boosts pancreatic cancer development (18). Approximately 5-10\% of the population are Lewis antigen negative and have no or scarce secretion of CA19-9 (10). Therefore, it is reasonable to infer that Lewis-negative pancreatic cancer is associated with lower levels of CA19-9 secretion. CA19-9 is not recommended as a biomarker for Lewis-negative pancreatic cancer (11). In the present study, $11.7 \%$ of patients with pancreatic cancer were Lewis negative. However, 24\% of Lewis-negative pancreatic cancer patients had high secretion of CA19-9 (>37 U/ml), which was also been reported by previous studies $(9,11,20)$. Therefore, the potential mechanisms should be explored.

Lewis gene plays an important role in the fucosylation of proteins, which catalyzes the reaction of adding fucose to the $\alpha 1-3,4$ position $(21,22)$. Several studies have shown that the Lewis gene is an oncogene that could accelerate cancer development $(21,23)$. Silencing of Lewis by shRNA could reduce the expression of Lewis antigens and therefore decrease the adhesion abilities of cancer cells to endothelial cells with E-selectin expression (21). Theoretically, Lewis-negative pancreatic cancer, which has Lewis gene dysfunction and fucosylation deficiency, is supposed to be an indolent subgroup for the role of the Lewis gene in boosting cancer development. Interestingly, in the present study, Lewis-negative pancreatic cancer was shown to be an aggressive subgroup of pancreatic cancer with special clinical and molecular features, which may be explained by the fact that fucosylation is an important biological process, and fucosylation deficiency affects both cancer development and human body physiology.

MUC16, also known as CA125, is a membrane bound mucin that belongs to the glycoprotein family (24). Fucosylation is an essential process for MUC16 biosynthesis. MUC16 is an important biomarker for the diagnosis of various types of cancer, such as ovarian and digestive cancers $(11,15,25)$. MUC16 could also be applied in the management of pancreatic cancer, including diagnosis, predicting resectability, monitoring therapeutic response and follow-up (11). Importantly, several studies have reported that MUC16 could promote cancer progression $(24,26)$. A study has shown that MUC16 could mediate cell-cell adhesion by affecting the E-cadherin/ $\beta$-catenin complex (26). In our previous study, MUC16 was shown to promote pancreatic cancer progression by Foxp3 expression and tumor-associated Treg enrichment through the activation of the IL-6-JAK2/STAT3 pathway (24). In the present study, Lewis-negative pancreatic cancer was shown to have higher levels of MUC16 secretion than its counterpart. The molecular mechanism explaining the association of the Lewis gene and MUC16 biosynthesis and the effect of MUC16 high secretion on cancer development undoubtedly deserve further research.

CaPan-1 was confirmed by sequencing to be a Lewis antigen-negative cell line. However, CaPan-1 presented properties similar to Lewis antigen-positive cell lines, including low proliferation rate, low migration ability and high level of fucosylation. These findings indicate that heterogeneity even exists in the Lewis-negative subgroup.

The present study is restricted by only presenting clinicopathological and molecular features of Lewis-negative pancreatic cancer. The potential mechanisms accounting for the aggressive properties of Lewis-negative pancreatic cancer should be investigated. In addition, the clinical value of the identification of Lewis-negative pancreatic cancer in guiding clinical practice should be further explored. Efforts should also be paid to the reasons Lewis-negative pancreatic cancers have Lewis antigen expression. Finally, the reasons for CaPan-1, a Lewis-negative pancreatic cancer cell line, having characteristics different from other Lewis-negative pancreatic cancer cell lines should also be investigated.

\section{Acknowledgements}

Not applicable.

\section{Funding}

The study was supported by the National Natural Science Foundation of China (grant nos. 81625016, 81871940 and 81902417), the Shanghai Natural Science Foundation (grant 
no. 17ZR1406300), the Shanghai Cancer Center Foundation for Distinguished Young Scholars (grant no. YJJQ201803), and the Fudan University Personalized Project for 'Double Top' Original Research (grant no. XM03190633).

\section{Availability of data and materials}

The datasets used and/or analyzed during the current study are available from the corresponding author on reasonable request.

\section{Authors' contributions}

$\mathrm{CL}, \mathrm{KJ}$ and SD performed the experiments and the scientific literature search, and contributed to the figures and the writing of the manuscript. All authors participated in the data analysis and reviewed the manuscript. GL and XY conceived and designed the study and wrote the manuscript. All authors read and approved the final manuscript.

\section{Ethics approval and consent to participate}

The study protocol was authorized by the Ethics Committee of the Fudan University Shanghai Cancer Center (Shanghai, China). Written informed consent was acquired from all of the patients enrolled. All animal procedures were approved by the Institutional Animal Care Committee of Fudan University (Shanghai, China).

\section{Patient consent for publication}

Not applicable.

\section{Competing interests}

The authors declare that they have no competing interests.

\section{References}

1. Siegel RL, Miller KD and Jemal A: Cancer statistics, 2019. CA Cancer J Clin 69: 7-34, 2019.

2. Ryan DP, Hong TS and Bardeesy N: Pancreatic adenocarcinoma. N Engl J Med 371: 2140-2141, 2014.

3. Long J, Luo GP, Xiao ZW, Liu ZQ, Guo M, Liu L, Liu C, Xu J, Gao YT, Zheng Y, et al: Cancer statistics: Current diagnosis and treatment of pancreatic cancer in Shanghai, China. Cancer Lett 346: 273-277, 2014.

4. Wallace DR, Spandidos DA, Tsatsakis A, Schweitzer A, Djordjevic V and Djordjevic AB: Potential interaction of cadmium chloride with pancreatic mitochondria: Implications for pancreatic cancer. Int J Mol Med 44: 145-156, 2019.

5. Suzuki K, Takeuchi O, Suzuki Y and Kitagawa Y: Mechanisms of metformin's anti tumor activity against gemcitabine resistant pancreatic adenocarcinoma. Int J Oncol 54: 764-772, 2019.

6. Luo G, Liu C, Guo M, Long J, Liu Z, Xiao Z, Jin K, Cheng H, Lu Y, Ni Q, et al: CA19-9-Low\&Lewis (+) pancreatic cancer: A unique subtype. Cancer Lett 385: 46-50, 2017.

7. Robert M, Jarlier M, Gourgou S, Desseigne F, Ychou M, Bouché O, Juzyna B, Conroy T and Bennouna J: Retrospective analysis of CA19-9 decrease in patients with metastatic pancreatic carcinoma treated with FOLFIRINOX or gemcitabine in a randomized phase III study (ACCORD11/PRODIGE4). Oncology 93: 367-376, 2017.

8. Humphris JL, Chang DK, Johns AL, Scarlett CJ, Pajic M, Jones MD, Colvin EK, Nagrial A, Chin VT, Chantrill LA, et al; NSW Pancreatic Cancer Network: The prognostic and predictive value of serum CA19.9 in pancreatic cancer. Ann Oncol 23: 1713-1722, 2012.
9. Luo G, Fan Z, Cheng H, Jin K, Guo M, Lu Y, Yang C, Fan K, Huang Q, Long J, et al: New observations on the utility of CA19-9 as a biomarker in Lewis negative patients with pancreatic cancer. Pancreatology 18: 971-976, 2018

10. Guo M, Luo G, Lu R, Shi W, Cheng H, Lu Y, Jin K, Yang C, Wang Z, Long J, et al: Distribution of Lewis and Secretor polymorphisms and corresponding CA19-9 antigen expression in a Chinese population. FEBS Open Bio 7: 1660-1671, 2017.

11. Luo G, Liu C, Guo M, Cheng H, Lu Y, Jin K, Liu L, Long J, Xu J, Lu R, et al: Potential biomarkers in Lewis negative patients with pancreatic cancer. Ann Surg 265: 800-805, 2017.

12. Gao HF, Wang QY, Zhang K, Chen LY, Cheng CS, Chen H, Meng ZQ, Zhou SM and Chen Z: Overexpressed $N$-fucosylation on the cell surface driven by FUT3, 5, and 6 promotes cell motilities in metastatic pancreatic cancer cell lines. Biochem Biophys Res Commun 511: 482-489, 2019.

13. Luo G, Guo M, Jin K, Liu Z, Liu C, Cheng H, Lu Y, Long J, Liu L, Xu J, et al: Optimize CA19-9 in detecting pancreatic cancer by Lewis and Secretor genotyping. Pancreatology 16: 1057-1062, 2016.

14. Wiśniewski JR, Zougman A, Nagaraj N and Mann M: Universal sample preparation method for proteome analysis. Nat Methods 6: 359-362, 2009.

15. Luo G, Xiao Z, Long J, Liu Z, Liu L, Liu C, Xu J, Ni Q and Yu X: CA125 is superior to CA19-9 in predicting the resectability of pancreatic cancer. J Gastrointest Surg 17: 2092-2098, 2013.

16. Tsai S, George B, Wittmann D, Ritch PS, Krepline AN, Aldakkak M, Barnes CA, Christians KK, Dua K, Griffin M et al: Importance of normalization of CA19-9 levels following neoadjuvant therapy in patients with localized pancreatic cancer. Ann Surg: Oct 11, 2018 (Epub ahead of print).

17. Takada A, Ohmori K, Yoneda T, Tsuyuoka K, Hasegawa A, Kiso M and Kannagi R: Contribution of carbohydrate antigens sialyl Lewis A and sialyl Lewis X to adhesion of human cancer cells to vascular endothelium. Cancer Res 53: 354-361, 1993.

18. Engle DD, Tiriac H, Rivera KD, Pommier A, Whalen S, Oni TE, Alagesan B, Lee EJ, Yao MA, Lucito MS, et al: The glycan CA19-9 promotes pancreatitis and pancreatic cancer in mice. Science 364: 1156-1162, 2019.

19. GebauerF, Wicklein D, StübkeK, Nehmann N,Schmidt A,Salamon J, Peldschus K, Nentwich MF, Adam G, Tolstonog G, et al: Selectin binding is essential for peritoneal carcinomatosis in a xenograft model of human pancreatic adenocarcinoma in pfp--/rag2-- mice. Gut 62: 741-750, 2013.

20. Hamada E, Taniguchi T, Baba S and Maekawa M: Investigation of unexpected serum CA19-9 elevation in Lewis-negative cancer patients. Ann Clin Biochem 49: 266-272, 2012.

21. Padró M, Cobler L, Garrido M and de Bolós C: Down-regulation of FUT3 and FUT5 by shRNA alters Lewis antigens expression and reduces the adhesion capacities of gastric cancer cells. Biochim Biophys Acta 1810: 1141-1149, 2011.

22. Orntoft TF, Vestergaard EM, Holmes E, Jakobsen JS, Grunnet N, Mortensen M, Johnson P, Bross P, Gregersen N, Skorstengaard K, et al: Influence of Lewis alpha1-3/4-L-fucosyltransferase (FUT3) gene mutations on enzyme activity, erythrocyte phenotyping, and circulating tumor marker sialyl-Lewis a levels. J Biol Chem 271: 32260-32268, 1996.

23. Weston BW, Hiller KM, Mayben JP, Manousos GA, Bendt KM, Liu R and Cusack JC Jr: Expression of human alpha(1,3)fucosyltransferase antisense sequences inhibits selectin-mediated adhesion and liver metastasis of colon carcinoma cells. Cancer Res 59: 2127-2135, 1999.

24. Fan K, Yang C, Fan Z, Huang Q, Zhang Y, Cheng H, Jin K, Lu Y, Wang Z, Luo G, et al: MUC16 C terminal-induced secretion of tumor-derived IL- 6 contributes to tumor-associated Treg enrichment in pancreatic cancer. Cancer Lett 418: 167-175, 2018.

25. Fleming ND, Cass I, Walsh CS, Karlan BY and Li AJ: CA125 surveillance increases optimal resectability at secondary cytoreductive surgery for recurrent epithelial ovarian cancer. Gynecol Oncol 121: 249-252, 2011.

26. Akita K, Tanaka M, Tanida S, Mori Y, Toda M and Nakada H: CA125/MUC16 interacts with Src family kinases, and over-expression of its C-terminal fragment in human epithelial cancer cells reduces cell-cell adhesion. Eur J Cell Biol 92: 257-263, 2013.

This work is licensed under a Creative Commons Attribution-NonCommercial-NoDerivatives 4.0 International (CC BY-NC-ND 4.0) License. 